\title{
FANO MANIFOLDS WITH WEAK ALMOST KÄHLER-RICCI SOLITONS
}

\author{
FENG WANG AND XIAOHUA ZHU*
}

\begin{abstract}
In this paper, we prove that a sequence of weak almost Kähler-Ricci solitons under further suitable conditions converge to a Kähler-Ricci soliton with complex codimension of singularities at least 2 in the Gromov-Hausdorff topology. As a corollary, we show that on a Fano manifold with the modified K-energy bounded below, there exists a sequence of weak almost Kähler-Ricci solitons which converge to a Kähler-Ricci soliton with complex codimension of singularities at least 2 in the Gromov-Hausdorff topology.
\end{abstract}

\section{Contents}

0. Introduction

1. A version of pseudolocality theorem 2

2. A Ricci curvature estimate 10

3. Estimate for the distance functions 14

4. Almost Kähler Ricci solitons $\quad 19$

References 24

\section{INTRODUCTION}

In [WZ], we studied the structure of the limit space for a sequence of Riemannian manifolds with the Bakry-Émery Ricci curvature bounded below in the Gromov-Hausdorff topology. In particular, for a sequence of weak almost Kähler-Ricci solitons $\left\{\left(M_{i}, g^{i}, J_{i}\right)\right\}$, we showed that there exists a subsequence of $\left\{\left(M_{i}, g^{i}, J_{i}\right)\right\}$ which converge to a metric space $\left(Y, g_{\infty}\right)$ with complex codimension of singularities at least 2 in the Gromov-Hausdorff topology. As in $[\mathrm{CC}]$ for Riemannian manifolds with the Ricci curvature bounded below, each tangent space on $\left(Y, g_{\infty}\right)$ is a metric cone. The present paper is a continuance of [WZ]. We further prove the smoothness of the

1991 Mathematics Subject Classification. Primary: 53C25; Secondary: 53C55, 58J05.

Key words and phrases. Bakry-Émery Ricci curvature, Gromov-Hausdorff topology, Kähler-Ricci flow, almost weak solitons.

* Partially supported by the NSFC Grants 10990013 and 11271022. 
metric $g_{\infty}$ on the regular part $\mathcal{R}$ of $Y$ under further suitable conditions. Actually, $g_{\infty}$ is a Kähler-Ricci soliton on $\mathcal{R}$.

Inspired by a recent work of Tian and Wang on weak almost Einstein metrics [TW], we use the Kähler-Ricci flow to smooth the sequence of Kähler metrics $\left\{\left(M_{i}, g^{i}, J_{i}\right)\right\}$ to get the $C^{\infty}$-convergence. To realize this, we shall first establish a version of Perelman's pseudolocality theorem for the Hamilton's Ricci flow with the Bakerly-Émergy Ricci curvature condition, then we control the deformation of distance functions along the Kähler-Ricc flow as in $[\mathrm{TW}]$.

It is useful to mention that there are two new ingredients in our case compared to $[\mathrm{TW}]$ : One is that we modify the Kähler-Ricci flow to derive an estimate for the modified Ricci curvature (cf. Section 2); another is that we estimate the growth of the $C^{0}$-norm of holomorphic vector fields associated to the Kähler-Ricci solitons along the flow (cf. Section 4). The late is usually dependent of the initial metric $g^{i}$ of the Kähler-Ricci flow. But for a family of Kähler metrics $g^{s}(0<s<1)$ constructed from solutions of a family of complex Monge-Ampère equations on a Fano manifold with the modified K-energy bounded below [WZ], we get a uniform $C^{0}$-norm for the holomorphic vector field (cf. Lemma 4.8) under the deformed metrics.

The following can be regarded as the main result in this paper.

Theorem 0.1. Let $(M, J)$ be a Fano manifold with the modified K-energy bounded below. Then there exists a sequence of weak almost Kähler-Ricci solitons on $(M, J)$ which converge to a Kähler-Ricci soliton with complex codimension of singularities at least 2 in the Gromov-Hausdorff topology. In the other words, a Fano manifold with the modified K-energy bounded below can be deformed to a Kähler-Ricci soliton with complex codimension of singularities at least 2 .

The organization of paper is as follows. In Section 1, we prove a pseudolocality theorem of Perelman for the Hamilton's Ricci flow under the BakryÉmery Ricci curvature condition. In Section 2, we focus on the (modified) Kähler-Ricc flow to give a local estimate for the Ricci curvature along the flow. Section 3 is devoted to estimate the distance functions along the Kähler-Ricc flow. In Section 4, we prove the main theorems in this paper, Theorem 4.2 and Theorem 4.5 (Theorem 0.1).

\section{A VERSiOn OF PSEUdolocality THEOREM}

In this section, we prove a version of Perelman's pseudolocality theorem with Bakerly-Émergy Ricci curvature condition (cf. Theorem 11.2 in [Pe]). A similar version was recently appeared in $[\mathrm{TW}]$. Since our case is lack of 
the lower bound of scalar curvature, in particular the lower bound of Ricci curvature, we will modify the arguments both in [Pe] and [TW].

First we recall a result about an estimate of isoperimetric constant on a geodesic ball. This result comes essentially from a lemma in [Li] and the volume comparison theorem with the Bakerly-Émergy Ricci curvature bounded below in [WW].

Lemma 1.1. Let $(M, g)$ be a Riemannian manifold with

$$
\operatorname{Ric}(g)+\text { hess }{ }_{g} f \geq-(n-1) c g,\left|\nabla_{g} f\right| \leq A .
$$

Then for any geodesic balls in $M,\left(B_{p}(s)\right),\left(B_{p}(r)\right)$ with $r \geq s$, there exists a uniform $C=C(n)$ such that

$$
I D_{\frac{n}{n-1}}\left(B_{p}(s)\right) \geq C^{\frac{1}{n}}\left(\frac{\operatorname{vol}\left(B_{p}(r)\right)-\operatorname{vol}\left(B_{p}(s)\right)}{v(r+s)}\right)^{\frac{n+1}{n}},
$$

where $v(r)=e^{2 A r} \operatorname{vol}_{c}(r)$ and $\operatorname{vol}_{c}(r)$ denotes the volume of $r$-geodesic ball in the space form with constant curvature $-c$.

Lemma 1.1 will be used to get a uniform Sobolev constant in the proof of following pseudolocality theorem in the Bakerly-Émergy geometry.

Theorem 1.2. For any $\alpha, r \in[0,1]$, there exist $\tau=\tau(n, \alpha), \eta=\eta(n, \alpha), \epsilon=$ $\epsilon(n, \alpha), \delta=\delta(n, \alpha)$, such that if $\left(M^{n}, g(\cdot, t)\right)\left(0 \leq t \leq(\epsilon r)^{2}\right)$ is a solution of Ricci flow,

$$
\frac{\partial g}{\partial t}=-2 \operatorname{Ric}(g)
$$

whose initial metric $g(\cdot, 0)=g_{0}$ satisfies

$$
\operatorname{Ric}\left(g_{0}\right)+\text { hess }_{g_{0}} f \geq-(n-1) r^{-2} \tau^{2} g_{0},|\nabla f|_{g_{0}} \leq r^{-1} \eta,
$$

and

$$
\operatorname{vol}\left(B_{q}\left(r, g_{0}\right)\right) \geq(1-\delta) c_{n} r^{n},
$$

where $c_{n}$ is the volume of unit ball in the Euclidean space $\mathbb{R}^{n}$, then for any $x \in B_{q}\left(\epsilon r, g_{0}\right)$ and $t \in\left(0,(\epsilon r)^{2}\right]$, we have

$$
|R m(x, t)|<\alpha t^{-1}+(\epsilon r)^{-2},
$$

Moreover,

$$
\operatorname{vol} B_{x}(\sqrt{t}) \geq \kappa(n) t^{\frac{n}{2}}
$$

where $\kappa(n)$ is a uniform constant. 
Proof. By scaling the metric, we may assume $r=1$ in the theorem. As in $[\mathrm{Pe}]$, we use the argument by contradiction to prove (1.6). On contrary, we suppose that for some $\alpha>0$, there are $\tau_{i}, \eta_{i}, \delta_{i}, \epsilon_{i}$ which approaching zero as $i \rightarrow \infty$, and there are a sequence of manifolds $\left\{\left(M_{i}, g^{i}\right)\right\}$ which satisfying (1.4) and (1.5) with some points $q_{i} \in M_{i}$ such that (1.6) doesn't hold at $\left(x_{i}, \bar{t}_{i}\right)$ for some points $x_{i} \in B_{q_{i}}\left(\epsilon_{i}, g_{0}^{i}\right)$ some time $\bar{t}_{i} \leq \epsilon_{i}^{2}$ along the Ricci flows $\left(M_{i}, g_{t}^{i}=g^{i}(\cdot, t)\right)$ with $g^{i}=g_{0}^{i}$ as the initial metrics. Without the loss of generality, we may also assume that

$$
|R m(x, t)| \leq \alpha t^{-1}+\left(\epsilon_{i}\right)^{-2}, \forall t \in\left(0, \bar{t}_{i}\right], x \in B_{q_{i}}\left(\epsilon_{i}, g_{0}^{i}\right) .
$$

Then as showed in $[\mathrm{Pe}]$, for any $A<\frac{1}{100 n \epsilon_{i}}$, there exist points $\left(\bar{x}_{i}, \bar{t}_{i}\right)$ such that for any $(x, t)$ with

$$
\begin{gathered}
\bar{t}_{i}-\frac{1}{2} \alpha Q^{-1} \leq t \leq \bar{t}_{i}, d_{g_{t}^{i}}\left(x, \bar{x}_{i}\right) \leq \frac{1}{10} A Q^{-\frac{1}{2}}, \\
|R m(x, t)| \leq 4 Q
\end{gathered}
$$

where $Q=\left|R m\left(\bar{x}_{i}, \bar{t}_{i}\right)\right| \rightarrow \infty$.

Now we consider a solution $u_{i}(x, t)=\left(4 \pi\left(\bar{t}_{i}-t\right)\right)^{-\frac{n}{2}} e^{-p_{i}(t, x)}$ of the conjugate heat equation associated to the flow $\left(M_{i}, g_{t}^{i}\right)$ which starts from a delta function $\delta\left(\bar{x}_{i}, \bar{t}_{i}\right)$. Namely, $u_{i}(x, t)$ satisfies

$$
\square^{*} u_{i}(x, t)=\left(-\frac{\partial}{\partial t}-\Delta+R\right) u_{i}(x, t)=0,
$$

where $R=R(\cdot, t)$ is the scalar curvature of $g_{t}^{i}$. Then the function

$$
v_{i}(x, t)=\left[\left(\bar{t}_{i}-t\right)\left(2 \Delta p_{i}-\left|\nabla p_{i}\right|^{2}+R\right)+p_{i}-n\right] u_{i}
$$

is nonpositive. Moreover, there exists a positive constant $\beta$ such that

$$
\int_{B_{\bar{x}_{i}}\left(\sqrt{\bar{t}_{i}-\tilde{t}_{i}}, g_{\tilde{t}_{i}}^{i}\right)} v_{i} \leq-\beta,
$$

for some $\tilde{t}_{i} \in\left[\bar{t}-\frac{1}{2} \alpha Q^{-1}, \bar{t}_{i}\right]$, when $i$ is large enough [Pe].

Let $\phi$ be a cut-off function which is equal to 1 on $[0,1]$ and decreases to 0 on $[1,2]$. Moreover, it satisfies $\phi^{\prime \prime} \geq-10 \phi,\left(\phi^{\prime}\right)^{2} \leq 10 \phi$. Putting $h_{i}=\phi\left(\frac{\tilde{d}_{i}(x, t)}{10 A \sqrt{\bar{t}_{i}}}\right)$, where $\tilde{d}_{i}(x, t)=d_{g_{t}^{i}}\left(\bar{x}_{i}, x\right)+200 n \sqrt{t}$. Then by Lemma 8.3 in [Pe] with the help of (1.8), we get

$$
\begin{aligned}
\left(\frac{\partial}{\partial t}-\Delta\right) h_{i} & =\frac{1}{10 A \sqrt{\bar{t}_{i}}}\left(d_{t}-\Delta d+\frac{100 n}{\sqrt{\bar{t}}}\right) \phi^{\prime}-\left(\frac{1}{10 A \sqrt{\bar{t}_{i}}}\right)^{2} \phi^{\prime \prime} \\
& \leq\left(\frac{1}{10 A \sqrt{\bar{t}_{i}}}\right)^{2} 10 \phi, \forall t \in\left(0, \bar{t}_{i}\right], x \in B_{q}\left(\epsilon, g_{0}^{i}\right) .
\end{aligned}
$$


It follows

$$
\begin{aligned}
& \frac{d}{d t} \int_{M_{i}}\left(-h_{i} v_{i}\right)=\int_{M_{i}} \square h_{i}\left(-v_{i}\right)+\int_{M_{i}} h_{i} \square^{*} v_{i} \\
& \leq-\frac{1}{100 A^{2} \bar{t}_{i}} \int_{M_{i}} h_{i} v_{i},
\end{aligned}
$$

where $\square=\frac{\partial}{\partial t}-\Delta$ and we used the fact that $\square^{*} v_{i} \leq 0[\mathrm{Pe}]$. Thus by (1.10), we obtain

$$
\beta\left(1-A^{-2}\right) \leq-\int_{M_{i}}\left(h_{i} v_{i}\right)(0, \cdot) .
$$

Similarly, we can show

$$
\int_{M_{i}}\left(\hat{h}_{i} u_{i}\right)(\cdot, 0) \geq 1-4 A^{-2}
$$

where $\hat{h}_{i}=\phi\left(\frac{\tilde{d}_{i}(x, t)}{5 A \sqrt{\bar{t}_{i}}}\right)$. The above implies that

$$
\int_{B_{\bar{x}_{i}}\left(20 A \sqrt{\bar{t}_{i}}\right) \backslash B_{\bar{x}_{i}}\left(10 A \sqrt{\bar{t}_{i}}\right)} u_{i}(\cdot, 0) \leq 1-\int_{M_{i}} \hat{h}_{i} u_{i} \leq 4 A^{-2} .
$$

On the other hand, by (1.13), we see that

$$
\begin{aligned}
& -\int_{M_{i}}\left(h_{i} v_{i}\right)=\int_{M}\left[\bar{t}_{i}\left(-2 \Delta p_{i}+\left|\nabla p_{i}\right|^{2}-R\right)-p_{i}+n\right] h_{i} u_{i} \\
& =\int_{M_{i}}\left[-\bar{t}_{i}\left|\nabla \tilde{p}_{i}\right|^{2}-\tilde{p}_{i}+n\right] \tilde{u}_{i}+\int_{M_{i}}\left[\bar{t}_{i}\left(\frac{\left|\nabla h_{i}\right|^{2}}{h_{i}}-R h_{i}\right)-h_{i} \ln h_{i}\right] u_{i} \\
& \leq \int_{M_{i}}\left[-\bar{t}_{i}\left|\nabla \tilde{p}_{i}\right|^{2}-\tilde{p}_{i}+n\right] \tilde{u}_{i}-\bar{t}_{i} \int_{M_{i}} R \tilde{u}_{i}+A^{-2}+100 \epsilon^{2},
\end{aligned}
$$

where $\tilde{u}_{i}=h_{i} u_{i}$ and $\tilde{p}_{i}=p_{i}-\ln h_{i}$. Note that $R+\Delta f_{i} \geq-(n-1)$. Then

$$
\begin{array}{r}
-\int_{M_{i}} R \tilde{u}_{i} \leq n-1-\int_{M_{i}}\left\langle\nabla f_{i}, \tilde{u}_{i}\right\rangle \leq n-1+\eta_{i} \int_{M_{i}}\left|\nabla \tilde{u}_{i}\right| \\
\leq n-1+\eta_{i} \sqrt{\int_{M_{i}}\left|\nabla \tilde{p}_{i}\right|^{2} \tilde{u}_{i}} \leq n+\eta_{i} \int_{M_{i}}\left|\nabla \tilde{p}_{i}\right|^{2} \tilde{u}_{i} .
\end{array}
$$

Hence, by (1.11), we get

$$
\int_{M_{i}}\left[-\bar{t}_{i}\left(1-\eta_{i}\right)\left|\nabla \tilde{p}_{i}\right|^{2}-\tilde{p}_{i}+n\right] \tilde{u}_{i} \geq \beta\left(1-A^{-2}\right)-(100+n) \epsilon^{2}-A^{-2} .
$$

Therefore, by rescaling these metrics $g_{0}^{i}$ to $\widehat{g_{0}^{i}}=\frac{1}{2}\left[\bar{t}_{i}\left(1-\eta_{i}\right)\right]^{-1} g_{0}^{i}$, we derive

$$
\int_{B_{\bar{x}_{i}}(20 A)}\left[-\frac{1}{2}\left|\nabla \tilde{p}_{i}\right|^{2}-\tilde{p}_{i}+n\right] \widehat{u}_{i} \geq\left(1-\eta_{i}\right)^{\frac{n}{2}} \mu>\mu_{0}>0,
$$


where $\widehat{u}_{i}=(2 \pi)^{-\frac{n}{2}} e^{-\tilde{p}_{i}}$ and $\mu=\beta\left(1-A^{-2}\right)-(100+n) \epsilon^{2}-A^{-2}$. Normalize $\widehat{u}_{i}$ by multiplying a constant $c$ so that

$$
\int_{B_{\bar{x}_{i}}(20 A)} c \widehat{u}_{i}=1
$$

By (1.12), it is easy to see that (1.14) still holds for the normalized $\widehat{u}_{i}$.

Next as in $[\mathrm{TW}]$. we introduce a functional

$$
F_{i}(u)=\int_{B_{\bar{x}_{i}}(20 A)}\left(2|\nabla u|^{2}-2 u^{2} \log u-n(1+\log \sqrt{2 \pi}) u^{2}\right),
$$

defined for any nonnegative functions $u \in W_{0}^{1,2}\left(B_{\bar{x}_{i}}(20 A), \widehat{g_{0}^{i}}\right)$ with

$$
\int_{B_{\bar{x}_{i}}(20 A)} u^{2}=1 \text {. }
$$

Clearly, by (1.14), we have

$$
\lambda_{i} \leq F_{i}\left(\sqrt{c \widehat{u}_{i}}\right) \leq-\mu_{0}<0,
$$

where $\lambda_{i}=\inf _{u \in W_{0}^{1,2}\left(B_{\bar{x}_{i}}(20 A), \widehat{g_{0}^{i}}\right)} F_{i}(u)$. According to [Ro], the infinity of $F_{i}(u)$ can be achieved by a minimizer $\phi_{i}$ which satisfies the Euler-Lagrange equation on $\left(B_{\overline{x_{i}}}(20 A), \widehat{g_{0}^{i}}\right)$,

$$
-2 \Delta \phi_{i}(x)-2 \phi_{i}(x) \log \phi_{i}(x)-n(1+\log \sqrt{2 \pi}) \phi_{i}(x)=\lambda_{i} \phi_{i}(x) .
$$

We need to estimate the $L^{\infty}$-norms and gradient norms of those $\phi_{i}$. Note that $\log x \leq \frac{n}{2} x^{\frac{2}{n}}$. Then

$$
\begin{aligned}
& \lambda_{i}+n(1+\log \sqrt{2 \pi}) \\
& =2 \int_{B_{\bar{x}_{i}}(20 A)}\left|\nabla \phi_{i}(x)\right|^{2}-2 \int_{B_{\bar{x}_{i}}(20 A)} \phi_{i}(x)^{2} \log \phi_{i}(x) \\
& \geq 2 \int_{B_{\bar{x}_{i}}(20 A)}\left|\nabla \phi_{i}(x)\right|^{2}-n \int_{B_{\bar{x}_{i}}(20 A)} \phi_{i}(x) \phi_{i}(x)^{\frac{n+2}{n}} \\
& \geq 2 \int_{B_{\bar{x}_{i}}(20 A)}\left|\nabla \phi_{i}(x)\right|^{2}-n\left(\int_{B_{\bar{x}_{i}}(20 A)} \phi_{i}(x)^{\left.\frac{2 n}{n-2}\right)^{\frac{n-2}{2 n}}}\right. \\
& \geq 2 \int_{B_{\bar{x}_{i}}(20 A)}\left|\nabla \phi_{i}(x)\right|^{2}-\left\{a ^ { 2 } \left(\int_{B_{\bar{x}_{i}}(20 A)} \phi_{i}(x)^{\left.\left.\frac{2 n}{n-2}\right)^{\frac{n-2}{2 n}}+\frac{n^{2}}{4 a^{2}}\right\} .}\right.\right.
\end{aligned}
$$

Since the Sobolev constants $C_{S}$ are uniformly bounded below on $\left(B_{\bar{x}}\left(\frac{1}{2}\right)\right.$, $g_{0}^{i}$ ) according to Lemma 1.1, by choosing the number $a$ small enough, we see that $\lambda_{i}$ is uniformly bounded below and $\int_{B_{\bar{x}_{i}}(20 A)}\left|\nabla \phi_{i}(x)\right|^{2}$ is uniformly bounded. Applying the standard Moser iteration method to (1.16), we will get

$$
\left|\phi_{i}(x)\right|<C_{1}\left(\mu_{0}, n, C_{S}\right) .
$$


As a consequence, $\phi_{i}(x)$ is an almost sub-solution of the Lapalace equation. Hence, we can also get a uniform oscillation estimate for $\phi_{i}(x)$ near the boundary of $B_{\bar{x}_{i}}(20 A)$. In fact, as in [WT], we can show that for any $w \in$ $\partial B_{\bar{x}_{i}}(20 A)$

$$
O s c_{B_{w}\left(2^{-N}\right)}\left(\phi_{i}\right)<C \gamma^{N-1}+\frac{\gamma^{N-1}-4^{-N+1}}{4(4 \gamma-1)},
$$

for some uniform $C$, where $N \geq 2$ is any integer and the number $\gamma$ can be chosen in the interval $\left(\frac{1}{2}, 1\right)$.

To get the interior gradient estimate for $\phi_{i}(x)$, we will also use the Moser iteration method. For simplicity, we let $\phi=\phi_{i}$ for each $i$. First we note that by (1.16) and the estimate (1.17), it holds

$$
\langle\nabla \phi, \nabla \Delta \phi\rangle \geq-C_{2}\left(\nu, n, C_{S}\right)|\nabla \phi(x)|^{2} .
$$

Then by the Bochner identity,

$$
\frac{1}{2} \Delta|\nabla \phi|^{2}=\mid \text { hess }\left.\phi\right|^{2}+R_{i j} \phi_{i} \phi_{j}+\langle\nabla \phi, \nabla \Delta \phi\rangle,
$$

we obtain

$$
\frac{1}{2} \Delta|\nabla \phi|^{2} \geq \mid \text { hess }\left.\phi\right|^{2}-f_{i j} \phi_{i} \phi_{j}-\left(C_{2}+(n-1) \tau^{2}\right)|\nabla \phi|^{2} .
$$

Let $\rho$ be a cut-off function on the interval $[0,20 A]$ which is supported in a subset of $[0,20 A s)$, where $s<1$. Then multiplying both sides of (1.20) by $\rho\left(d\left(\bar{x}_{i},.\right) w^{p}\right.$, where $w=|\nabla \phi|^{2}$ and $p \geq 0$, we get

$$
\begin{aligned}
& \frac{2 p}{(p+1)^{2}} \int_{B_{\bar{x}_{i}}(20 A)} \rho\left(d\left(\bar{x}_{i}, .\right)\right)\left|\nabla w^{\frac{p+1}{2}}\right|^{2} \\
& =\frac{1}{2} \int_{B_{\bar{x}_{i}}(20 A)} \rho\left(d\left(\bar{x}_{i}, .\right)\right)(-\Delta w) w^{p}-\frac{1}{2} \int_{B_{\bar{x}_{i}}(20 A)}\left\langle\nabla \rho\left(d\left(\bar{x}_{i}, .\right)\right), \nabla w\right\rangle w^{p} \\
& \leq \int_{B_{\bar{x}_{i}}(20 A)} \rho\left(d\left(\bar{x}_{i}, .\right)\right) w^{p}\left(-\mid \text { hess }\left.\phi\right|^{2}+f_{i j} \phi_{i} \phi_{j}+C_{2}|\nabla \phi|^{2}\right)
\end{aligned}
$$

$$
-\frac{1}{2} \int_{B_{\bar{x}_{i}}(20 A)} w^{p}\left\langle\nabla \rho\left(d\left(\bar{x}_{i}, .\right)\right), \nabla w\right\rangle .
$$

On the other hand, using the integration by parts, we have

$$
\begin{aligned}
& \int_{B_{\bar{x}_{i}}(20 A)} \rho\left(d\left(\bar{x}_{i}, .\right)\right) w^{p} f_{i j} \phi_{i} \phi_{j} \\
& =-\int_{B_{\bar{x}_{i}}(20 A)} \rho_{l} f_{l} \phi_{i} \phi_{j} w^{p}+\int_{B_{\bar{x}_{i}}(20 A)} \rho f_{j} \phi_{i j} \phi_{j} w^{p} \\
& -p \int_{B_{\bar{x}_{i}}(20 A)} \rho w^{p-1} f_{i} w_{j} \phi_{i} \phi_{j}-\int_{B_{\bar{x}_{i}}(20 A)} \rho w^{p} f_{i} \phi_{i} \Delta \phi .
\end{aligned}
$$


Observe that

$$
\begin{aligned}
\left|\int_{B_{\bar{x}_{i}}(20 A)} \rho_{l} f_{l} \phi_{i} \phi_{j} w^{p}\right| & \leq \eta \int_{B_{\bar{x}_{i}}(20 A)}\left|\rho^{\prime}\right| w^{p+1}, \\
\left|\int_{B_{\bar{x}_{i}}(20 A)} \rho f_{j} \phi_{i j} \phi_{j} w^{p}\right| & \leq 2 \eta \int_{B_{\bar{x}_{i}(20 A)} \rho\left(\mid \text { hess }\left.\phi\right|^{2}+w\right) w^{p},} \rho \int_{\int_{B_{\bar{x}_{i}}(20 A)} \rho w^{p-1} f_{i} w_{j} \phi_{i} \phi_{j} \mid} \leq 2 \eta\left(\int_{B_{\bar{x}_{i}}(20 A)} \rho w^{p-1}|\nabla w|^{2}+\int_{B_{\bar{x}_{i}}(20 A)} \rho w^{p+1}\right), \\
\left|\int_{B_{\bar{x}_{i}(20 A)}} \rho w^{p} f_{i} \phi_{i} \Delta \phi\right| & \leq C_{3}\left(\nu_{0}, n, C_{s}\right) \eta \int_{B_{\bar{x}_{i}}(20 A)} \rho w^{p+\frac{1}{2}} .
\end{aligned}
$$

Hence, by (1.21), we get

$$
\begin{aligned}
& \frac{p}{(p+1)^{2}} \int_{B_{\bar{x}_{i}}(20 A)} \rho\left(d\left(\bar{x}_{i}, .\right)\right)\left|\nabla w^{\frac{p+1}{2}}\right|^{2} \\
& \leq C_{4} \int_{B_{\bar{x}_{i}}(20 A)}\left(\rho+p \eta \rho+\rho^{\prime}\right) w^{p+1}+C_{5} \int_{B_{\bar{x}_{i}}(20 A)} \eta \rho w^{p+\frac{1}{2}} .
\end{aligned}
$$

Since we may assume that $w \geq 1$, we deduce

$$
\begin{aligned}
& \frac{p}{(p+1)^{2}} \int_{B_{\bar{x}_{i}}(20 A)} \rho(d(\bar{x}, .))\left|\nabla w^{\frac{p+1}{2}}\right|^{2} \\
& \leq C_{5}^{\prime} \int_{B_{\bar{x}_{i}}(20 A)}\left(\rho+p \eta \rho+\rho^{\prime}\right) w^{p+1}, \forall p \geq 0 .
\end{aligned}
$$

Note that the Sobolev constants are uniformly bounded below on $\left(B_{\bar{x}_{i}}(20 A)\right.$, $\left.\widehat{g_{0}^{i}}\right)$. Therefore, by choosing the suitable cut-off functions $\eta$ in (1.22), we use the iteration method to derive

$$
\left\|\nabla \phi_{i}\right\|_{C^{0}\left(B_{\bar{x}_{i}}(20 s A)\right)}^{2} \leq C_{6}\left(1+\int_{B_{\bar{x}_{i}}(20 A)}\left|\nabla \phi_{i}\right|^{2}\right)<C .
$$

It remains to analyze the limit of $\phi_{i}$. According to Corollary 4.8 in [WZ], we see that $\left(M_{i}, \widehat{g_{0}^{i}}\right)$ converge to the euclidean space $\mathbb{R}^{n}$ in the GromovHausdorff topology. Thus by the estimates (1.17) and (1.23), there exists a subsequence of $\phi_{i}$ which converge to a continuous limit $\phi_{\infty} \geq 0$ on the standard $B_{0}(20 A) \subset \mathbb{R}^{n}$.

Claim 1.3. $\phi_{\infty}$ is a solution of the following equation on $B_{0}(20 A)$,

$$
-2 \Delta \phi_{\infty}-2 \phi_{\infty} \log \phi_{\infty}-n(1+\log \sqrt{2 \pi}) \phi_{\infty}=\lambda_{\infty} \phi_{\infty}
$$

where $\lambda_{\infty}<0$.

As in [TW], to prove (1.24), it suffices to show that

$$
-\phi_{\infty}=\int_{B_{0}(20 A)} G(z, y)\left(\frac{\lambda_{\infty}+n(1+\log \sqrt{2 \pi})}{2}+\log \phi_{\infty}\right) \log \phi_{\infty} .
$$


Here $G(z, y)$ is the Green function on the ball $B_{0}(20 A)$, which is given by

$$
G(z, y)=\frac{1}{(n-2) n c_{n}}\left(d^{2-n}(z, y)-d^{2-n}(0, z) d^{2-n}\left(z^{*}, y\right)\right)
$$

where $z^{*}$ is the conjugate point of $z$.

Choose a sequence $z_{k} \rightarrow z, z_{k}^{*} \rightarrow z^{*}$. By the Laplacian comparison for the distance functions on $\left(B_{\overline{x_{i}}}(20 A), \widehat{g_{0}^{i}}\right)[\mathrm{WW}]$,

$$
\begin{aligned}
& \Delta d\left(z_{k}, .\right) \leq(n-1) \tau_{i} \operatorname{coth} \tau_{i} d\left(z_{k}, .\right)+2 \eta_{i} \\
& \leq \frac{n-1}{d\left(z_{k}, .\right)}+(n-1) \tau_{i}+2 \eta_{i}
\end{aligned}
$$

we have

$$
\Delta d^{2-n}\left(z_{k}, .\right)+(n-2) d^{1-n}\left(z_{k}, .\right)\left((n-1) \tau_{i}+2 \eta_{i}\right) \geq 0
$$

It follows

$$
\begin{aligned}
& \int_{B_{z_{k}}(20 A) \backslash z_{k}}\left|\Delta d^{2-n}\left(z_{k}, .\right)\right| \\
& \leq \int_{B_{z_{k}}(20 A) \backslash\left\{z_{k}\right\}}\left|\Delta d^{2-n}\left(z_{k}, .\right)+(n-2) d^{1-n}\left(z_{k}, .\right)\left((n-1) \tau_{i}+2 \eta_{i}\right)\right|
\end{aligned}
$$

$$
+\int_{B_{z_{k}}(20 A) \backslash\left\{z_{k}\right\}}(n-2) d^{1-n}\left(z_{k}, .\right)\left((n-1) \tau_{i}+2 \eta_{i}\right) .
$$

By a direct computation, we obtain

$$
\int_{B_{z_{k}}(20 A) \backslash\left\{z_{k}\right\}}\left|\Delta d^{2-n}\left(z_{k}, .\right)\right| \rightarrow 0, \text { as } k \rightarrow \infty .
$$

Note that

$$
\begin{aligned}
& \int_{B_{z_{k}}(20 A)} d^{2-n}\left(z_{k}, y\right) \Delta \phi_{k}(y)= \\
& (n-2) n c_{n} \phi_{k}\left(z_{k}\right)+\int_{B_{x_{k}}(20 A) \backslash\left\{z_{k}\right\}} \phi_{k}(y) \Delta d^{2-n}\left(z_{k}, y\right) .
\end{aligned}
$$

Hence we derive that

$$
\lim _{k \rightarrow \infty} \int_{B_{z_{k}}(20 A)} d^{2-n}\left(z_{k}, y\right) \Delta \phi_{k}(y)=(n-2) n c_{n} \phi_{\infty}(z) .
$$

Similarly, since $z_{k}^{*}$ is outside $B_{x_{k}}(20 A)$, we have

$$
\lim _{k \rightarrow \infty} \int_{B_{z_{k}}(20 A)} d^{2-n}\left(z_{k}^{*}, y\right) \Delta \phi_{k}(y)=0 .
$$


Combining (1.27) and (1.28), we get

$$
\begin{aligned}
& -\phi_{\infty}(z) \\
& =-\lim _{k \rightarrow \infty} \int_{B_{z_{k}}(20 A)}\left(d^{2-n}\left(z_{k}, y\right)-d^{2-n}\left(x_{k}, z_{k}\right) d^{2-n}\left(z_{k}^{*}, y\right)\right) \Delta \phi_{k}(y) \\
& =\lim _{k \rightarrow \infty} \int_{B_{z_{k}}(20 A)}\left(d^{2-n}\left(z_{k}, y\right)-d^{2-n}\left(x_{k}, z_{k}\right) d^{2-n}\left(z_{k}^{*}, y\right)\right) \\
& \times\left(\frac{\lambda_{k}+n(1+\log \sqrt{2 \pi})}{2}+\log \phi_{k}\right) \phi_{k} \\
& =\int_{B_{0}(20 A)} G(z, y)\left(\frac{\lambda_{\infty}+n(1+\log \sqrt{2 \pi})}{2}+\log \phi_{\infty}\right) \phi_{\infty} .
\end{aligned}
$$

The claim is proved.

By the estimates (1.18), $\phi_{\infty}$ is in fact in $C_{0}\left(B_{0}(20 A)\right)$. Thus by (1.24), we get

$$
F\left(\phi_{\infty}\right)=\int_{B_{0}(20 A)}\left(2\left|\nabla \phi_{\infty}\right|^{2}-2 \phi_{\infty}^{2} \log \phi_{\infty}-n(1+\log \sqrt{2 \pi}) \phi_{\infty}^{2}\right)=\lambda_{\infty}<0
$$

which is a contradiction to the Log-Sobolev inequality in $\mathbb{R}^{n}[\mathrm{Gr}]$. The proof of (1.6) is completed.

To obtain (1.7), it suffices to estimate the lower bound of the injective radius at $x$. This can be done by using the same blowing-up argument as in the proof of (1.6) (cf. [Pe], [TW]). We leave it to the readers.

\section{A Ricci Curvature estimate}

In this section, we prove several technical lemmas which will be used in next sections. From now on we assume that $M$ is an $n$-dimensional Fano manifold with a reductive holomorphic vector field $X$ [TZ1]. As in [TZ3], we consider the following modified Kähler-Ricci flow,

$$
\frac{\partial}{\partial t} g=-\operatorname{Ric}(g)+g+L_{X} g
$$

with a $K_{X}$-invariant initial Kähler metric $g_{0}$ in $2 \pi c_{1}(M)$, where $K_{X}$ is the one-parameter compact subgroup generated by $\operatorname{im}(X)$. Thus $L_{X} g$ is a real valued complex hessian tensor. If we scale $g_{0}$ by $\frac{1}{\lambda}$, where $0<\lambda \leq 1$, then (2.1) becomes

$$
\frac{\partial}{\partial t} g=-\operatorname{Ric}(g)+\lambda g+\lambda L_{X} g
$$

Clearly, the flow is solvable for any $t>0$ and $\omega_{g_{t}} \in \frac{2 \pi}{\lambda} c_{1}(M)$, where $g_{t}=$ $g(\cdot, t)$. 
By a direct computation from the flow (2.2), we see that

$$
\begin{aligned}
\frac{\partial}{\partial t} R_{i \bar{j}}= & \Delta R_{i \bar{j}}-R_{i \bar{k}} R_{k \bar{j}}+R_{l \bar{k}} R_{i \bar{j} k \bar{l}} \\
& -\lambda \Delta \theta_{i \bar{j}}+\frac{\lambda}{2}\left(R_{i \bar{k}} \theta_{k \bar{j}}+R_{k \bar{j}} \theta_{i \bar{k}}\right)-\lambda R_{i \bar{j} k l} \theta_{l \bar{k}}
\end{aligned}
$$

and

$$
\frac{\partial}{\partial t} \theta_{i \bar{j}}=L_{X}\left(-\operatorname{Ric}(g)+\lambda g+\lambda L_{X} g\right),
$$

where $\theta=\theta_{g_{t}}$ is a potential of $X$ associated to $g_{t}$ such that $\theta_{i \bar{j}}=L_{X} g_{t}$. Thus if we let $H=\operatorname{Ric}(g)-\lambda g-\lambda L_{X} g$, then we have

$$
\frac{\partial}{\partial t} H=\Delta H+\lambda L_{X} H+\Lambda(H, R m)
$$

where $\Lambda$ is a linear operator with bounded coefficients with respect to the metric $g_{t}$ and $R m=R m(\cdot, t)$ is the sectional curvature of $g_{t}$.

Moreover, we have

\section{Lemma 2.1.}

$$
\begin{aligned}
& \frac{\partial}{\partial t}(R-\lambda \Delta \theta-n \lambda) \\
& =\lambda(R-\lambda \Delta \theta-n \lambda)+\Delta(R-\lambda \Delta \theta-n \lambda)-\lambda \Delta \frac{\partial}{\partial t} \theta \\
& +|\operatorname{Ric}(g)-\lambda g-\lambda \sqrt{-1} \partial \bar{\partial} \theta|^{2} .
\end{aligned}
$$

The following lemma is a consequence of Theorem 1.2 in Section 1.

Lemma 2.2. Let $g=g_{t}$ be a solution of (2.2) with $\omega_{g_{0}} \in \frac{2 \pi}{\lambda} c_{1}(M)$. Suppose that there exists a small $\delta \leq \delta_{0}<<1$ such that $g_{0}$ satisfies:

$$
\begin{aligned}
& \text { i) } \operatorname{Ric}\left(g_{0}\right)+\lambda L_{X} g_{0} \geq-(n-1) \delta^{2} g_{0} \\
& \text { ii) }|X|_{g_{0}}(x) \leq \frac{\delta}{\lambda}, \forall x \in B_{q}\left(1, g_{0}\right) \\
& \text { iii) } \operatorname{vol}\left(B_{q}\left(1, g_{0}\right)\right) \geq(1-\delta) c_{n} .
\end{aligned}
$$

Then

$$
|R m(x, t)| \leq 4 t^{-1}, \forall x \in B_{q}\left(\frac{3}{4}, g_{0}\right), t \in(0,2 \delta]
$$

and

$$
\operatorname{vol}\left(B_{x}(\sqrt{t}, g(t))\right) \geq \kappa(n) t^{n},
$$

where $\kappa=\kappa(n)$ is a uniform constant.

By Lemma 2.1 and Lemma 2.2, we prove 
Lemma 2.3. Let $g=g_{t}$ be a solution of (2.2) with $\omega_{g_{0}} \in \frac{2 \pi}{\lambda} c_{1}(M)$. Suppose that for any $t \in[-2,1]$ (we may replace $t$ by $t-3$ ), $g_{t}$ satisfies:

i) $\operatorname{inj}\left(q, g_{t}\right) \geq 1$;

ii) $|R m(x, t)| \leq 1$ and $|X|_{g_{t}} \leq \frac{A}{\lambda}, \forall x \in B_{q}\left(1, g_{t}\right)$.

Then

$$
\begin{aligned}
& \left|\operatorname{Ric}(g)-\lambda g-\lambda L_{X} g\right|(q, 0) \\
& \leq C(A, n)\left\{\int_{-2}^{1} d t \int_{M}|R-n \lambda-\Delta \theta| \omega_{g_{t}}^{n}\right\}^{\frac{1}{2}}
\end{aligned}
$$

Proof. Putting $h=|H|$, by (2.4), we get

$$
\left(\frac{\partial}{\partial t}-\Delta\right) h \leq \frac{\Lambda_{1}(H, H, R m)}{h}+\lambda X(h)+\lambda \frac{\Lambda_{2}(\sqrt{-1} \partial \bar{\partial} \theta, H, H)}{h},
$$

where $\Lambda_{1}, \Lambda_{2}$ are two linear operators with bounded coefficients with respect to the metric $g_{t}$. Note that under the conditions i) and ii) in the lemma the Sobolev constants are uniformly bounded below on $B_{q}\left(\frac{1}{2}, g_{0}\right)$. Then using the method of Moser iteration, we obtain

$$
\begin{aligned}
& \left|\operatorname{Ric}(g)-\lambda g-\lambda L_{X} g\right|(q, 0) \\
& \leq C(A, n)\left\{\int_{-1}^{0} d t \int_{M}\left|\operatorname{Ric}(g)-\lambda g-\lambda L_{X} g\right|^{2} \omega_{g_{t}}^{n}\right\}^{\frac{1}{2}}
\end{aligned}
$$

On the other hand, we see that there exist some $t_{1} \in[-2,-1]$ and $t_{2} \in[0,1]$ such that

$$
\begin{gathered}
\int_{M}|R-\lambda \Delta \theta-n \lambda| \omega_{g_{t_{1}}}^{n} \leq \int_{-2}^{-1} d t \int_{M}|R-\lambda \Delta \theta-n \lambda| \omega_{g_{t}}^{n}, \\
\int_{M}|R-\lambda \Delta \theta-n \lambda| \omega_{g_{t_{2}}}^{n} \leq \int_{0}^{1} d t \int_{M}|R-\lambda \Delta \theta-n \lambda| \omega_{g_{t}}^{n} .
\end{gathered}
$$

Then integrating (2.1) in Lemma 2.1, it follows

$$
\begin{aligned}
& \int_{t_{1}}^{t_{2}} d t \int_{M}\left|\operatorname{Ric}(g)-\lambda g-\lambda L_{X} g\right|^{2} \omega_{g_{t}}^{n} \\
& \leq \int_{t_{1}}^{t_{2}} d t \int_{M}|R-\lambda \Delta \theta-n \lambda| \omega_{g_{t}}^{n} \\
& +\int_{M}|R-\lambda \Delta \theta-n \lambda| \omega_{g_{t_{1}}}^{n}+\int_{M}|R-\lambda \Delta \theta-n \lambda| \omega_{g_{t_{2}}}^{n} \\
& \leq 3 \int_{-2}^{1} d t \int_{M}|R-\lambda \Delta \theta-n \lambda| \omega_{g_{t}}^{n} .
\end{aligned}
$$


Hence by (2.8), we derive

$$
\begin{aligned}
h(q, 0) & \leq C(A, m)\left\{\int_{t_{1}}^{t_{2}} d t \int_{M}|R-\lambda \Delta \theta-n \lambda| \omega_{g_{t}}^{n}\right\}^{\frac{1}{2}} \\
& \leq 3 C(A, m)\left\{\int_{-2}^{1} d t \int_{M}|R-\lambda \Delta \theta-n \lambda| \omega_{g_{t}}^{n}\right\}^{\frac{1}{2}} .
\end{aligned}
$$

Lemma 2.4. Under the conditions of Lemma 2.2 and $|X|_{g_{t}} \leq \frac{A}{\lambda \sqrt{t}}$, we have

$$
\begin{aligned}
& \left|\operatorname{Ric}(g)-\lambda g-\lambda L_{X} g\right|(x, s) \\
& \leq C(n, A) s^{-\frac{n+2}{2}}\left\{\int_{0}^{2 s} d t \int|R-n \lambda-\lambda \Delta \theta| \omega_{g_{t}}^{n}\right\}^{\frac{1}{2}},
\end{aligned}
$$

for $0<s \leq \delta$.

Proof. By Lemma [2.2, we know that for $x \in B_{q}\left(\frac{3}{4}, g_{0}\right)$ and $t \in(0,2 \delta]$,

$$
|R m(x, t)| \leq t^{-1} \text { and } \operatorname{vol}\left(B_{x}(\sqrt{t})\right) \geq \kappa(n) t^{n} .
$$

Then the injective radius estimate in $[\mathrm{CGT}]$ implies that

$$
\operatorname{inj}(x, t) \geq \xi(n) \sqrt{t} .
$$

Let $l=\xi(n)^{-1} s^{-\frac{1}{2}}$. By scaling the metric $g_{t}$ as

$$
\tilde{g}_{t}=l^{2} g\left(l^{-2} t+s\right), t \in[-2,1],
$$

$\tilde{g}_{t}$ satisfies

$$
\frac{\partial}{\partial t} \tilde{g}=-\operatorname{Ric}(\tilde{g})+\frac{\lambda}{l^{2}} \tilde{g}+\frac{\lambda}{l^{2}} L_{X} \tilde{g} .
$$

Moreover, $\tilde{g}_{t}$ satisfies the conditions i) and ii) in Lemma 2.3 for any $t \in$ $[-2,1]$ while $\lambda$ is replaced by $\frac{\lambda}{l^{2}}$.

Note that

$$
|X|_{\tilde{g}_{t}}=l|X|_{g} \leq \frac{2 C l}{\lambda \sqrt{s}}=\frac{2 C \xi(n) l^{2}}{\lambda} .
$$

Applying Lemma 2.3 to $\tilde{g}_{t}$, we have

$$
\begin{aligned}
& \left|\operatorname{Ric}(\tilde{g})-\frac{\lambda}{l^{2}} \tilde{g}-\frac{\lambda}{l^{2}} L_{X} \tilde{g}\right|_{\tilde{g}}(x, 0) \\
& \leq C(n, A)\left\{\int_{-2}^{1} d t \int\left|R(\tilde{g})-n \frac{\lambda}{l^{2}}-\frac{\lambda}{l^{2}} \operatorname{tr}_{\tilde{g}}\left(L_{X} \tilde{g}\right)\right| \omega_{\tilde{g}_{t}}^{n}\right\}^{\frac{1}{2}}
\end{aligned}
$$

Observe that

$$
\left|\operatorname{Ric}(g)-\lambda g-\lambda L_{X} g\right|_{g}(x, s)=l^{2}\left|\operatorname{Ric}(\tilde{g})-\frac{\lambda}{l^{2}} \tilde{g}-\frac{\lambda}{l^{2}} L_{X} \tilde{g}\right|_{\tilde{g}}(x, 0)
$$


and

$$
\begin{aligned}
& \int_{s-l^{-2}}^{s+2 l^{-2}} d t \int|R-n \lambda-\lambda \Delta \theta| \omega_{g_{t}}^{n} \\
& =l^{-n} \int_{-2}^{1} d t \int\left|R(\tilde{g})-n \frac{\lambda}{l^{2}}-\frac{\lambda}{l^{2}} \operatorname{tr}_{\tilde{g}}\left(L_{X} \tilde{g}\right)\right| \omega_{\tilde{g}}^{n}
\end{aligned}
$$

Thus we get

$$
\begin{aligned}
& \left|\operatorname{Ric}(g)-\lambda g-\lambda L_{X} g\right|_{g}(x, s) \\
& \leq C(n, A) s^{-\frac{n+2}{2}}\left\{\int_{s-l^{-2}}^{s+2 l^{-2}} d t \int|R-n \lambda-\lambda \Delta \theta| \omega_{g_{t}}^{n}\right\}^{\frac{1}{2}},
\end{aligned}
$$

which implies (2.10).

\section{Estimate For the Distance FUnCtions}

We are going to compare the distance functions between the initial metric $g_{0}$ and $g_{\delta}$ in the flow (2.2). The following lemma is due to Perelman for the normalized Ricci flow $[\mathrm{Pe}]$.

Lemma 3.1. Let $g_{t}=g(\cdot, t)(0 \leq t \leq 1)$ be a solution of rescaled Ricci flow on $M^{n}$ (in our case, $M$ is Kähler),

$$
\frac{d}{d t} g=-\operatorname{Ric}(g)+\lambda g, g(0, \cdot)=g_{0},
$$

where $0<\lambda \leq 1$. Let $x_{1}, x_{2}$ be two points in $M$. Suppose that at time $t \geq 0$,

$$
\operatorname{Ric}\left(g_{t}\right)(x) \leq(2 n-1) K, \forall x \in B_{x_{1}}\left(r_{0}, g_{t}\right) \cup B_{x_{2}}\left(r_{0}, g_{t}\right)
$$

for some $r_{0}>0$. Then

$$
\frac{d}{d t} d_{g_{t}}\left(x_{1}, x_{2}\right) \geq \lambda d_{g_{t}}\left(x_{1}, x_{2}\right)-2(2 n-1)\left(\frac{2}{3} K r_{0}+r_{0}^{-1}\right) .
$$

Proof. Without loss of generality, we may assume that $t=0$. Putting

$$
\tilde{g}_{t}=(1-\lambda t) g\left(\frac{\log (1-\lambda t)}{-\lambda}\right),\left(0 \leq t<\frac{1}{\lambda}\right),
$$

then $\tilde{g}=\tilde{g}_{t}$ satisfies the Hamilton Ricci flow,

$$
\frac{\partial}{\partial t} \tilde{g}=-\operatorname{Ric}(\tilde{g})
$$

Since $\tilde{g}_{0}=g_{0}$, by applying Lemma 8.3 in $[\mathrm{Pe}]$, we have

$$
\left.\frac{d}{d t} d_{\tilde{g}_{t}}\right|_{t=0} \geq-2(2 n-1)\left(\frac{2}{3} K r_{0}+r_{0}^{-1}\right)
$$

Note that

$$
\tilde{d}_{t}=-\lambda d+d_{t}
$$

Hence (3.2) is true. 
By Lemma 3.1 together with Lemma 2.4 In Section 2, we give a lower bound estimate for the distance functions along the flow as follows.

Proposition 3.2. Under the assumption of Lemma 2.4, we have that for two points $x_{1}, x_{2}$ in $B_{q}\left(\frac{1}{2}, g_{0}\right)$,

$$
d_{g_{\delta}}\left(x_{1}, x_{2}\right) \geq d_{g_{0}}\left(x_{1}, x_{2}\right)-\frac{C_{0}}{\lambda}\left(\sqrt{t}+t^{-\frac{n}{2}} E^{\frac{1}{2}}\right), \forall t \in(0, \delta],
$$

where $C_{0}$ is a uniform constant and $E=\int_{0}^{2 \delta} d t \int_{M}|R-\lambda \Delta \theta-n \lambda| \omega_{g_{t}}^{n}$. In particular, when $E \leq \delta^{n+1}$,

$$
d_{g_{\delta}}\left(x_{1}, x_{2}\right) \geq d_{g_{0}}\left(x_{1}, x_{2}\right)-\frac{C_{0}}{\lambda} E^{\frac{1}{2(n+1)}} .
$$

Proof. Let $\Phi(t)$ be a one parameter subgroup generated by $\operatorname{real}(X)$. Then $\hat{g}_{t}=\Phi(-t)^{*} g_{t}$ is a solution of of the normalized flow (3.1). Applying Lemma 2.2 for two points $y_{1}=\Phi(-t) x_{1}$ and $y_{2}=\Phi(-t) x_{2}$ by choosing $r_{0}=\sqrt{t}$, together with Lemma 3.1 we have

It follows

$$
\frac{d}{d t} d_{\hat{g}_{t}}\left(y_{1}, y_{2}\right) \geq \frac{\lambda}{2} d_{\hat{g}_{t}}\left(y_{1}, y_{2}\right)-C_{1} t^{-\frac{1}{2}} .
$$

$$
d_{\hat{g}_{t}}\left(y_{1}, y_{2}\right) \geq d_{\hat{g}_{0}}\left(y_{1}, y_{2}\right)-2 C_{1} \sqrt{t}
$$

As a consequence, we derive

$$
\begin{aligned}
d_{g_{t}}\left(x_{1}, x_{2}\right) & =d_{\hat{g}_{t}}\left(y_{1}, y_{2}\right) \\
& \geq d_{g_{0}}\left(y_{1}, y_{2}\right)-2 C_{1} \sqrt{t} \\
& \geq d_{g_{0}}\left(x_{1}, x_{2}\right)-2\|X\|_{g_{0}} t-2 C_{1} \sqrt{t} \\
& \geq d_{g_{0}}\left(x_{1}, x_{2}\right)-\frac{C_{2} \sqrt{t}}{\lambda} .
\end{aligned}
$$

On the other hand, integrating (2.2), we get from Lemma 2.4.

$$
\begin{aligned}
& \log \frac{d_{g_{\delta}}\left(x_{1}, x_{2}\right)}{d_{g_{t}}\left(x_{1}, x_{2}\right)} \\
& \geq-C_{3} \int_{t}^{\delta} s^{-\frac{n+2}{2}} E^{\frac{1}{2}} d s \geq-C_{3}^{\prime} E^{\frac{1}{2}} t^{-\frac{n}{2}}, \forall t>0 .
\end{aligned}
$$

Hence combining (3.5) and (3.6), we obtain

$$
\begin{aligned}
& d_{g_{\delta}}\left(x_{1}, x_{2}\right) \geq d_{g_{t}}\left(x_{1}, x_{2}\right) e^{-C_{3}^{\prime} E^{\frac{1}{2}} t^{-\frac{n}{2}}} \\
& \geq\left(d_{g_{0}}\left(x_{1}, x_{2}\right)-\frac{C_{2} \sqrt{t}}{\lambda}\right) e^{-C_{3}^{\prime} E^{\frac{1}{2}} t^{-\frac{n}{2}}} \\
& \geq d_{g_{0}}\left(x_{1}, x_{2}\right)-\frac{C_{4}}{\lambda}\left(\sqrt{t}+t^{-\frac{n}{2}} E^{\frac{1}{2}}\right) .
\end{aligned}
$$

When $E \leq \delta^{n+1}$, we can choose $t=E^{\frac{1}{n+1}}$ to get (3.4). 
Next we use the above proposition to give an upper bound estimate for the distance functions by using a covering argument as in [TW].

Lemma 3.3. Let $(M, g(\cdot, t), q)(0 \leq t \leq 1)$ be a solution of (2.2) as in Lemma 2.4. Let $\Omega=B_{q}\left(1, g_{0}\right), \Omega^{\prime}=B_{q}\left(\frac{1}{2}, g_{0}\right)$. For every $l<\frac{1}{2}$, we define

$$
A_{+, l}=\sup _{B_{x}\left(s, g_{0}\right) \subset \Omega^{\prime}, s \leq l} c_{n}^{-1} s^{-2 n} \operatorname{vol}_{g_{0}}\left(B_{x}\left(s, g_{0}\right)\right)
$$

and

$$
A_{-, l}=\inf _{B_{x}\left(s, g_{\delta}\right) \subset \Omega^{\prime}, s \leq l} c_{n}^{-1} s^{-2 n} \operatorname{vol}_{g_{\delta}}\left(B_{x}\left(s, g_{\delta}\right)\right) .
$$

Then for any $x_{1}, x_{2} \in \Omega^{\prime \prime}=B_{q}\left(\frac{1}{4}, g_{0}\right)$, it holds

$$
d_{g_{\delta}}\left(x_{1}, x_{2}\right) \leq r+\frac{C_{0}}{\lambda} A_{+, 4 r}\left\{\left|\frac{A_{+, r}}{A_{-, r}}-1\right|^{\frac{1}{2 n}}+r^{-\frac{1}{2 n}} E^{\frac{1}{4 n(n+1)}}\right\} r,
$$

where $r=d_{g_{0}}\left(x_{1}, x_{2}\right) \leq \frac{1}{8}$ and $E<<r^{2(n+1)}$.

Proof. By Proposition 3.2, we see that

$$
B_{x_{1}}\left(r-\frac{C_{0}}{\lambda} E^{\frac{1}{2(n+1)}}, g_{\delta}\right) \subset B_{x_{1}}\left(r, g_{0}\right),
$$

where $C_{0}$ is the constance determined in (3.4). Then

$$
A_{-, r}\left(r-\frac{C_{0}}{\lambda} E^{\frac{1}{2(n+1)}}\right)^{2 n} \leq \operatorname{vol}_{g_{\delta}}\left(B_{x_{1}}\left(r-C_{0} E^{\frac{1}{2(n+1)}}, g_{\delta}\right)\right) .
$$

Let $s_{0}$ be the largest radius $s$ among all the balls $B_{x}\left(s, g_{0}\right)$ such that

$$
B_{x}\left(s, g_{0}\right) \subset B_{x_{1}}\left(r, g_{0}\right) \text { and } B_{x}\left(s, g_{0}\right) \cap B_{x_{1}}\left(r-\frac{C_{0}}{\lambda} E^{\frac{1}{2(n+1)}}, g_{\delta}\right)=\emptyset \text {. }
$$

Since the volume element $d \operatorname{vol}\left(g_{t}\right)$ satisfies

$$
\frac{d}{d t} d \operatorname{vol}\left(g_{t}\right)=(-R+n \lambda+\lambda \Delta \theta) d \operatorname{vol}\left(g_{t}\right),
$$

it is easy to see that there is a ball $B_{x_{0}}\left(s_{0}, g_{0}\right)$ such that

$$
\begin{aligned}
& \operatorname{vol}_{g_{\delta}}\left(B_{x_{0}}\left(s_{0}, g_{0}\right)\right) \\
& \leq \operatorname{vol}_{g_{\delta}}\left(B_{x_{1}}\left(r, g_{0}\right)\right)-\operatorname{vol}_{g_{\delta}}\left(B_{x_{1}}\left(r-\frac{C_{0}}{\lambda} E^{\frac{1}{2(n+1)}}, g_{\delta}\right)\right) \\
& \leq \operatorname{vol}_{g_{0}}\left(B_{x_{1}}\left(r, g_{0}\right)\right)-\operatorname{vol}_{g_{\delta}}\left(B_{x_{1}}\left(r-\frac{C_{0}}{\lambda} E^{\frac{1}{2(n+1)}}, g_{\delta}\right)\right)+E .
\end{aligned}
$$

Observe that

$$
B_{x_{0}}\left(s_{0}, g_{0}\right) \supseteq B_{x_{0}}\left(s_{0}-\frac{C_{0}}{\lambda} E^{\frac{1}{2(n+1)}}, g_{\delta}\right) .
$$

we have

$$
\begin{aligned}
A_{-, r}\left(s_{0}-\frac{C_{0}}{\lambda} E^{\frac{1}{2(n+1)}}\right)^{2 n} & \leq \operatorname{vol}_{g_{\delta}}\left(B_{x_{0}}\left(s_{0}-\frac{C_{0}}{\lambda} E^{\frac{1}{2(n+1)}}, g_{\delta}\right)\right) \\
& \leq \operatorname{vol}_{g_{\delta}}\left(B_{x_{0}}\left(s_{0}, g_{0}\right)\right) .
\end{aligned}
$$


Thus plugging the above inequality into (3.9) together with (3.8) and the fact that

$$
\operatorname{vol}_{g_{0}}\left(B_{x_{1}}\left(r, g_{0}\right)\right) \leq A_{+, r} r^{2 n}
$$

we obtain

$$
s_{0} \leq\left\{\left|\frac{A_{+, r}}{A_{-, r}}-1\right|+\frac{C_{0}}{\lambda} r^{-1} E^{\frac{1}{2(n+1)}}\right\}^{\frac{1}{2 n}} r+\frac{C_{0}}{\lambda} E^{\frac{1}{2(n+1)}} .
$$

On the other hand, since

$$
B_{x_{2}}\left(3 s_{0}, g_{0}\right) \cap B_{x_{1}}\left(r-\frac{C_{0}}{\lambda} E^{\frac{1}{2(n+1)}}, g_{\delta}\right) \neq \emptyset,
$$

we see that there exists some point

$$
x_{3} \in B_{x_{2}}\left(3 s_{0}, g_{0}\right) \cap B_{x_{1}}\left(r-\frac{C_{0}}{\lambda} E^{\frac{1}{2(n+1)}}, g_{\delta}\right) .
$$

Claim 3.4. There is a uniform constant $C_{1}=C_{1}(n)$ such that

$$
d_{g_{\delta}}\left(x_{2}, x_{3}\right) \leq C_{1} A_{+, 4 r} \max \left\{s_{0}, \frac{3 C_{0}}{\lambda} E^{\frac{1}{2(n+1)}}\right\} .
$$

Combining (3.11) with (3.10), we will finish the proof of (3.7) because of the triangle inequality

$$
d_{g_{\delta}}\left(x_{1}, x_{2}\right) \leq d_{g_{\delta}}\left(x_{1}, x_{3}\right)+d_{g_{\delta}}\left(x_{2}, x_{3}\right) .
$$

To prove Claim 3.4, we first assume that

$$
s_{0}>\frac{3 C_{0}}{\lambda} E^{\frac{1}{2(n+1)}} \text {. }
$$

Let $\gamma$ be the minimizing geodesic curve which connecting $x_{2}$ and $x_{3}$ in $\left(M, g_{0}\right)$. Choose $N$ geodesic balls $B_{z_{i}}\left(s_{0}, g_{\delta}\right)$ in $\left(M, g_{\delta}\right)$ such that $B_{z_{i}}\left(\frac{s_{0}}{2}, g_{\delta}\right)$ are disjoint. Since

$$
\begin{aligned}
& B_{z_{i}}\left(\frac{r_{0}}{2}, g_{\delta}\right) \subset B_{z_{i}}\left(\frac{s_{0}}{2}+\frac{C_{0}}{\lambda} E^{\frac{1}{2(n+1)}}, g_{0}\right) \\
& \subset B_{z_{i}}\left(s_{0}, g_{0}\right) \subset B_{x_{2}}\left(4 s_{0}, g_{0}\right) \subset B_{x_{1}}\left(\frac{1}{2}, g_{0}\right),
\end{aligned}
$$

we have

$$
\begin{aligned}
N A_{-, r}\left(\frac{s_{0}}{2}\right)^{2 n} & \leq \sum_{i=1}^{N} \operatorname{vol}_{g_{\delta}}\left(B_{z_{i}}\left(\frac{s_{0}}{2}, g_{\delta}\right)\right) \leq \operatorname{vol}_{g_{\delta}}\left(B_{x_{2}}\left(4 s_{0}\right)\right) \\
& \leq \operatorname{vol}_{g_{0}} B_{x_{2}}\left(4 s_{0}\right)+E \leq A_{+, 4 r}\left(4 s_{0}\right)^{2 n}+E
\end{aligned}
$$

Noticing that by the Bishop volume comparison and Lemma 2.2, we see that

$$
A_{-, r} \geq C(n, \delta)=C(n) .
$$

By (3.12), it follows

$$
N \leq C^{\prime} A_{+, 4 r}
$$


Since

$$
d_{g_{\delta}}\left(x_{2}, x_{3}\right) \leq 2 N s_{0}
$$

we deduce (3.11) from (3.10) immediately.

Secondly, we assume that

$$
s_{0} \leq \frac{3 C_{0}}{\lambda} E^{\frac{1}{2(n+1)}}
$$

In this case, we can copy the above argument of geodesic balls covering to prove (3.11) while the radius $s_{0}$ of balls is replaced by $\frac{3 C_{0}}{\lambda} E^{\frac{1}{2(n+1)}}$. The claim is proved.

Proposition 3.5. Let $(M, g(\cdot, t), q)(0 \leq t \leq 2 \delta)$ be a solution of (2.2) as in Lemma 2.4. Then for two points $x_{1}, x_{2} \in \Omega^{\prime \prime}=B_{q}\left(\frac{1}{4}, g_{0}\right)$ with $r=$ $d\left(x_{1}, x_{2}, g_{0}\right) \leq \frac{1}{8}$, we have

$$
d\left(x_{1}, x_{2}, g_{\delta}\right) \leq r+\frac{C_{0}}{\lambda} E^{\frac{1}{6 n(n+1)}} r
$$

if $E<<r^{6(n+1)}$.

Proof. By the Bishop volume comparison and Lemma 2.2, we see that

$$
A_{-, r} \geq 1-A r
$$

for some uniform constant $A$, where $r \leq \delta<<1$. Also by the volume comparison in $[\mathrm{WW}]$, we have

$$
A_{+, r} \leq 1+A r^{2}, \forall r \leq 1
$$

Applying Lemma 3.3 to any two points $x_{1}, x_{2} \in \Omega^{\prime \prime}$ with $d_{g_{0}}\left(x_{1}, x_{2}\right)=r \leq$ $\delta<<1$, we get

$$
d_{g_{\delta}}\left(x_{1}, x_{2}\right) r^{-1} \leq 1+\frac{C_{0}}{\lambda}\left(r^{\frac{1}{n}}+r^{-\frac{1}{2 n}} E^{4 n(n+1)}\right)
$$

For general two points $x_{1}, x_{2}$ with $d\left(x_{1}, x_{2}, g_{0}\right)=l \leq \frac{1}{8}$, we divide the minimal geodesic curve which connecting $x_{1}$ and $x_{2}$ into $N$ parts with the same length $\frac{l}{N} \leq \delta$. Thus by (3.14), we obtain

$$
\frac{d\left(x_{1}, x_{2}, g_{\delta}\right)}{N^{-1} l} \leq N\left\{1+\frac{C_{0}}{\lambda}\left\{\left(N^{-1} l\right)^{\frac{1}{n}}+\left(N^{-1} l\right)^{-\frac{1}{2 n}} E^{4 n(n+1)}\right\}\right\} .
$$

Choosing $N \sim l E^{-\frac{1}{6(n+1}}$, we derive (3.13). 


\section{Almost Kähler Ricci solitons}

In this section, we are able to prove the smoothness of the regular part of the limit space for a sequence of weak almost Kähler-Ricci solitons studied in [WZ]. Recall the definition of weak almost Kähler-Ricci solitons.

Definition 4.1. We call a sequence of Kähler metrics $\left\{\left(M_{i}, g^{i}, J_{i}\right)\right\}$ weak almost Kähler-Ricci solitons if there are uniform constants $\Lambda$ and $A$ such that
i) $\operatorname{Ric}\left(g^{i}\right)+L_{X_{i}} g^{i} \geq-\Lambda^{2} g^{i}, \quad i m\left(L_{X_{i}} g^{i}\right)=0$
ii) $\left|X_{i}\right|_{g^{i}} \leq A$
iii) $\lim _{i \rightarrow \infty}\left\|\operatorname{Ric}\left(g^{i}\right)-g^{i}+L_{X_{i}} g^{i}\right\|_{L_{M_{i}}^{1}\left(g^{i}\right)}=0$.

Here $\omega_{g^{i}} \in 2 \pi c_{1}\left(M_{i}, J_{i}\right)$ and $X_{i}$ are reductive holomorphic vector fields on Fano manifolds $\left(M_{i}, J_{i}\right)$.

We now assume that

$$
\operatorname{vol}_{g^{i}}\left(B_{p^{i}}(1)\right) \geq v>0, \text { for some } p^{i} \in M_{i} .
$$

Let $g_{t}^{i}=g^{i}(\cdot, t)$ be a solution of the Kähler-Ricci flow (2.1) on $\left(M_{i}, J_{i}\right)$ with $g^{i}$ the initial metric. Suppose that $g_{t}^{i}$ satisfies

$$
\left|X_{i}\right|_{g_{t}^{i}} \leq \frac{B}{\sqrt{t}}
$$

and

$$
\int_{0}^{1} d t \int_{M_{i}}\left|R\left(g_{t}^{i}\right)-\Delta \theta_{g_{t}^{i}}-n\right| \omega_{g_{t}^{i}}^{n} \rightarrow 0, \text { as } i \rightarrow \infty .
$$

Here $B$ is a uniform constant. We note that (4.2) and (4.3) have been used in Lemma 2.4, Proposition 3.2 and Proposition 3.5, respectively. Under the assumption (4.1)-(4.3), we prove

Theorem 4.2. Let $\left\{\left(M_{i}, g^{i}, J_{i}\right\}\right.$ be a sequence of weak almost Kähler-Ricci solitons. Suppose that $g^{i}$ satisfy the conditions (4.1)-(4.3). Then there exists a subsequence of $\left\{g^{i}\right\}$ which converge to a Kähler-Ricci soliton with complex codimension of singularities at least 2 in the Gromov-Hausdorff topology.

Proof. It was proved in [WZ] that under the condition (4.1) there exists a subsequence of $\left\{g^{i}\right\}$ which converge to a metric space $\left(Y, g_{\infty}\right)$ with complex codimension of singularities of $Y$ at least 2. Denote $\mathcal{R}$ as the regular part of $Y$. We want to show that $\mathcal{R}$ is an open manifold and $g_{\infty}$ is in fact a Kähler-Ricci soliton for some complex structure on $\mathcal{R}$. 
Let $y_{0} \in \mathcal{R}$. This means that the tangent cone $T_{y_{0}}$ at $y_{0}$ is isometric to $\mathbb{R}^{2 n}$. Then by the Volume Convergence Theorem 4.10 in [WZ], it is easy to see that for any $\delta>0$ there exists $r_{0}<<1$ such that

$$
\operatorname{vol}\left(B_{y_{0}}(r)\right)>(1-\delta) c_{n} r^{2 n}, \forall r<r_{0} .
$$

Again by the above convergence theorem together with the monotonicity of volume [WW], there exists an $\epsilon>0$ such that for any $y \in B_{y_{0}}\left(\epsilon, g_{\infty}\right)$ it holds

$$
\operatorname{vol}\left(B_{y}(r)\right)>(1-\delta) c_{n} r^{2 n}, \forall r<r_{0} .
$$

Claim 4.3. $y \in \mathcal{R}$ for any $y \in B_{y_{0}}\left(\epsilon, g_{\infty}\right)$.

For a fixed $r$, we choose a sequence of geodesic balls $B_{q_{i}}(r) \subset M_{i}$ which converge to $B_{y}(r)$ in the Gromov-Hausdorff topology. Then by (4.4), for $i$ large enough, we have

$$
\operatorname{vol}\left(B_{q_{i}}(r)\right)>(1-\delta) c_{n} r^{2 n}
$$

Scale $g^{i}$ to $\hat{g}^{i}=\frac{1}{r} g^{i}$ and we consider the solution $\hat{g}^{i}(\cdot, t)=\hat{g}_{t}^{i}$ of flow (2.2) with the initial metric $\hat{g}^{i}$, where $\lambda=r$. By applying Proposition 3.2 and Proposition 3.5 to each ball $B_{q_{i}}\left(1, \hat{g}^{i}\right)$, we obtain

$$
\left|d_{\hat{g}^{i}}\left(x_{1}, x_{2}\right)-d_{\hat{g}_{\delta}^{i}}\left(x_{1}, x_{2}\right)\right| \leq C E^{\frac{1}{6 n(n+1)}}, \forall x_{1}, x_{2} \in B_{q_{i}}\left(\frac{1}{4}, \hat{g}^{i}\right),
$$

where

$$
E=\frac{1}{r^{n-1}} \int_{0}^{2 \delta} d t \int_{M}\left|R\left(g_{t}^{i}\right)-\lambda \Delta \theta_{g_{t}^{i}}-n \lambda\right| \omega_{g_{t}^{i}}^{n} \rightarrow 0, \text { as } i \rightarrow \infty .
$$

On the other hand, since the curvature are uniformly bounded in $B_{q_{i}}\left(1, \hat{g}_{\delta}^{i}\right)$ by Lemma 2.2, $B_{q_{i}}\left(1, \hat{g}_{\delta}^{i}\right)$ converge to a smooth metric ball $B_{y \infty}\left(1, \hat{g}_{\infty}^{\prime}\right)$ by the regularity of $\hat{g}_{\delta}^{i}$. Hence by (4.6), we derive

$$
s^{-1} d_{G H}\left(B_{y}\left(s, g_{\infty}\right), B_{y \infty}\left(s, g_{\infty}^{\prime}\right)\right) \leq L s^{2}, \forall s \leq \frac{r}{4} .
$$

where $L$ is a uniform constant and $g_{\infty}^{\prime}=r \hat{g}_{\infty}$. This means that the tangent cone at $y$ is isometric to $\mathbb{R}^{2 n}$, so the claim is proved.

By the above claim, we see that there exists a small $r$ for any $y \in \mathcal{R}$ such that $B_{y}(r) \subset \mathcal{R}$ and (4.4) is satisfied. Then following the argument in the proof of Claim 4.3 , there exists a sequence of geodesic balls $\left(B_{q_{i}}(r), g_{\delta}^{i}\right) \subset M_{i}$ which converge to $B_{y}(r)$ in $C^{\infty}$-topology. Consequently, the potentials $\theta_{g_{\delta}^{i}}$ of $X_{i}$ restricted on $\left(B_{q_{i}}(r), g_{\delta}^{i}\right)$ converge to a smooth function $\theta_{\infty}$ defined on $B_{y}(r)$. Namely,

$$
\lim _{i \rightarrow \infty} \Psi_{i}^{*}\left(\theta_{g_{\delta}^{i}}\right)=\theta_{\infty}
$$


where $\Psi_{i}$ are diffeomorphisms from $B_{y}(r)$ to $B_{q_{i}}(r)$ such that $\Psi_{i}^{*}\left(g_{\delta}^{i}\right)$ converge to $g_{\infty}$ and $\Psi_{i}^{*} J_{i}$ converge to some limit complex structure $J_{\infty}$ on $B_{y}(r)$. By the regularity of flow (2.2) and the condition (4.3), $\theta_{\infty}$ satisfies in $B_{y}(r)$,

$$
\Delta \theta_{g_{\infty}}=R\left(g_{\infty}\right)-n \text { and } \partial \partial \theta_{\infty}=0 .
$$

Moreover, by (2.10) in Lemma 2.4, we get

$$
\operatorname{Ric}\left(g_{\infty}\right)-g_{\infty}-\sqrt{-1} \partial \bar{\partial} \theta_{\infty}=0, \text { in } B_{y}(r) .
$$

Hence, $\theta_{\infty}$ can be extended to a potential of holomorphic vector field $X_{\infty}$ on $\left(\mathcal{R}, J_{\infty}\right)$, and consequently $g_{\infty}$ is a Kähler-Ricci soliton on $\mathcal{R}$.

Remark 4.4. It seems that the limit space $Y$ in Theorem 4.2 is actually a normal algebraic variety as showed in recent papers by Tian, ChenDonaldson-Sun to solve the Yau-Tian-Donaldson conjecture for Kähler-Einstein metrics [T2], [CDS].

In [WZ], it was showed that there exists a sequence of weak almost KählerRicci solitons $g^{s}(s<1)$ on a Fano manifold $(M, g, J)$ if the modified Kenergy $\mu(\cdot)$ is bounded below. Here $\mu(\cdot)$ is defined for any $K_{X}$-invariant Kähler potential $\phi$ by $([\mathrm{TZ2}])$,

$$
\begin{aligned}
\mu(\phi) & =-\frac{n}{V} \int_{0}^{1} \int_{M} \dot{\psi}\left[\operatorname{Ric}\left(\omega_{\psi}\right)-\omega_{\psi}-\sqrt{-1} \partial \bar{\partial} \theta_{\omega_{\psi}}\right. \\
& \left.+\sqrt{-1} \bar{\partial}\left(h_{\omega_{\psi}}-\theta_{\omega_{\psi}}\right) \wedge \partial \theta \omega_{\psi}\right] \times e^{\theta_{\omega_{\psi}}} \omega_{\psi}^{n-1} \wedge d t .
\end{aligned}
$$

In fact, such $g^{s}$ are a family of Kähler metrics induced by the Kähler potential solutions $\phi_{s}$ of a family of complex Monge-Ampère equations, which are equivalent to a family of Ricci curvature equations,

$$
\operatorname{Ric}\left(\omega_{\phi_{s}}\right)=s \omega_{\phi_{s}}+(1-s) \omega_{g}+L_{X} \omega_{\phi_{s}} .
$$

(4.10) are also equivalent to equations,

$$
h_{\omega_{\phi_{s}}}-\theta_{\omega_{\phi_{s}}}=-(1-s) \phi_{s},
$$

where $h_{\omega_{\phi_{s}}}$ are the Ricci potentials of $\omega_{\phi_{s}}$.

In the following, we need to verify the conditions (4.2) and (4.3) for $g^{s}$. We note that (4.1) is true for $g^{s}$ [WZ]. Thus as an application of Theorem 4.2, we prove that

Theorem 4.5. There exists a sequence of weak almost Kähler-Ricci solitons $\left\{g^{s_{i}}\right\}\left(s_{i} \rightarrow 1\right)$ which converge to a Kähler-Ricci soliton with complex codimension of singularities at least 2 in the Gromov-Hausdorff topology. In the other words, a Fano manifold with the modified K-energy bounded below can be deformed to a Kähler-Ricci soliton with complex codimension of singularities at least 2. 


\section{Lemma 4.6.}

$$
h_{g_{s}}-\theta_{g_{s}} \rightarrow 0, \text { as } s \rightarrow 1
$$

Consequently,

$$
\left|h_{g_{s}}\right| \leq C \text {. }
$$

Proof. Recall the two functionals $I$ and $J$ defined for $K_{X}$-invariant Kähler potentials by ([Zh], [TZ1]),

$$
I(\phi)=\int_{M} \phi\left(e^{\theta_{\omega_{0}}} \omega_{0}^{n}-e^{\theta_{\omega}} \omega_{\phi}^{n}\right)
$$

and

$$
J(\phi)=\int_{0}^{1} \int_{M} \dot{\phi}_{t}\left(e^{\theta \omega_{0}} \omega_{0}^{n}-e^{\theta_{\omega_{\phi}}} \omega_{\phi}^{n}\right) d t .
$$

It was showed for the potential $\phi_{s}$ in [TZ1] that

$$
-\frac{d}{d s} \mu\left(\phi_{s}\right)=(1-s) \frac{d}{d s}(I-J)\left(\phi_{s}\right) .
$$

Then

$$
(I-J)\left(\phi_{s}\right)=-\frac{\mu\left(\phi_{s}\right)}{1-s}+\int_{0}^{s} \frac{\mu\left(\phi_{\tau}\right)}{(1-s)^{2}} d s .
$$

Since $\mu\left(\phi_{s}\right)$ is monotone and bounded below, $\lim _{s \rightarrow 1^{-}} \mu\left(\phi_{s}\right)$ exists. By 'Hôpital's rule, it is easy to see that

$$
\lim _{s \rightarrow 1^{-}}(1-s) \int_{0}^{s} \frac{\mu\left(\phi_{\tau}\right)}{(1-\tau)^{2}} d \tau=\lim _{s \rightarrow 1^{-}} \mu\left(\phi_{s}\right)
$$

Thus we get

$$
\lim _{s \rightarrow 1^{-}}(1-s)(I-J)\left(\phi_{s}\right)=0
$$

On the other hand, by using the Green formula [Ma] (also see [CTZ]), there exists a uniform constant $C$ such that

$$
\operatorname{osc}\left(\phi_{s}\right) \leq\left\|\phi_{s}\right\|_{C^{0}} \leq I\left(\phi_{s}\right)+C \text {. }
$$

It follows that

$$
(1-s)\left\|\phi_{s}\right\|_{C^{0}} \leq(1-s)\left(c(I-J)\left(\phi_{s}\right)+C\right) \rightarrow 0 \text { as } s \rightarrow 1 .
$$

Hence by (4.11), we obtain (4.12). (4.13) is a direct consequence of (4.12) since $\theta_{g_{s}}$ are uniformly bounded [Zh].

Lemma 4.7. Let $g_{t}^{s}=g^{s}(\cdot, t)$ be a solution of the Kähler-Ricci flow (2.1) with the above $g^{s}$ as an initial metric. Then

$$
|X|_{g_{t}^{s}} \leq \frac{B}{\sqrt{t}} .
$$


Proof. Let $u_{t}$ be the Kähler potential of $g_{t}^{s}$. Namely, it is defined by

$$
\omega_{g_{t}^{s}}=\omega_{g^{s}}+\sqrt{-1} \partial \bar{\partial} u
$$

According to Lemma 4.3 in [CTZ], we have

$$
\left|\nabla\left(\frac{\partial}{\partial t} u\right)\right|_{g_{t}^{s}} \leq e^{2} \frac{\left\|h_{g^{s}}-\theta_{g^{s}}\right\|_{C^{0}}}{\sqrt{t}}, 0<t \leq 1
$$

Since $\tilde{g}_{t}^{s}=\Phi_{t}^{*}\left(g_{t}^{s}\right)$ is a solution of the Kähler-Ricci flow,

$$
\frac{\partial}{\partial t} g=-\operatorname{Ric}(g)+g
$$

where $\Phi(-t)$ is an one parameter subgroup generated by real $(X)$, we also have for the Kähler potential $\tilde{u}$ of $\tilde{g}_{t}^{s}([\mathrm{~T} 1])$,

$$
\left|\nabla\left(\frac{\partial}{\partial t} \tilde{u}\right)\right|_{\tilde{g}_{t}^{s}} \leq e^{2} \frac{\left\|h_{g^{s}}\right\|_{C^{0}}}{\sqrt{t}}, \forall 0<t \leq 1
$$

Note that

$$
\frac{\partial}{\partial t} u=\Phi_{t}^{*}\left(\frac{\partial}{\partial t} \tilde{u}\right)+\theta_{g_{t}^{s}}+m(t)
$$

We get

$$
|X|_{g_{t}^{s}}=\left|\nabla \theta_{g_{t}^{s}}\right|_{g_{t}^{s}} \leq\left|\nabla\left(\frac{\partial}{\partial t} \tilde{u}\right)\right|_{\tilde{g}_{t}^{s}}+\left|\nabla\left(\frac{\partial}{\partial t} u\right)\right|_{g_{t}^{s}} .
$$

Now (4.14) follows from (4.13) immediately.

Lemma 4.8. Let $g_{t}^{s}=g^{s}(\cdot, t)$ be a solution of the Kähler-Ricci flow as in Lemma 4.7. Then

$$
\int_{0}^{1} d t \int_{M}\left|R\left(g_{t}^{s}\right)-\Delta \theta_{g_{t}^{s}}-n\right| \omega_{g_{t}^{s}}^{n} \rightarrow 0, \text { as } s \rightarrow 1 .
$$

Proof. First by (4.10), we note that

$$
(\Delta+X)\left(h_{g^{s}}-\theta_{g^{s}}\right) \geq-(1-s) n-(1-s)\left|X\left(\phi_{s}\right)\right| \geq-(1-s)\left(c_{1}+n\right),
$$

where $c_{1}=\sup \left\{\|X(\phi)\|_{C^{0}(M)} \mid K_{X}\right.$ - invariant Kähler potential $\left.\phi\right\}$ is a bounded number $[\mathrm{Zh}]$. By the Maximum Principle, it follows that (cf. Lemma 4.2 in $[\mathrm{CTZ}])$,

$$
(\Delta+X)\left(h_{g_{t}^{s}}-\theta_{g_{t}^{s}}\right) \geq-(1-s)\left(c_{1}+n\right) e^{t}, \forall 0<t .
$$

The above implies that (cf. Lemma 4.4 in [CTZ]),

$$
\begin{aligned}
& \int_{M}\left|\nabla\left(h_{g_{t}^{s}}-\theta_{g_{t}^{s}}\right)\right|^{2} e^{\theta_{g_{t}^{s}}} \omega_{g_{t}^{s}}^{n} \\
& \leq 2 e^{2}\left(c_{1}+n\right)(1-s)\left\|h_{g^{s}}-\theta_{g^{s}}\right\|_{C^{0}(M)}, \quad \forall 0<t \leq 1 .
\end{aligned}
$$


Hence by (4.14), we get

$$
\begin{aligned}
\int_{0}^{1} d t \int_{M}\left|X\left(h_{g_{t}^{s}}-\theta_{g_{t}^{s}}\right)\right| e^{\theta_{g_{t}^{s}}} \omega_{g_{t}^{s}}^{n} & \leq \int_{M}\left|\nabla\left(h_{g_{t}^{s}}-\theta_{g_{t}^{s}}\right)\right| e^{\theta_{g_{t}^{s}}} \omega_{g_{t}^{s}}^{n} \int_{0}^{1}|X|_{g_{t}^{s}} d t \\
& \leq C(1-s)^{\frac{1}{2}} \int_{0}^{1} \frac{1}{\sqrt{t}} d t \rightarrow 0, \text { as } s \rightarrow 1 .
\end{aligned}
$$

Therefore,

$$
\begin{aligned}
& \int_{0}^{1} d t \int_{M}\left|R\left(g_{t}^{s}\right)-\Delta \theta_{g_{t}^{s}}-n\right| e^{\theta_{g_{t}^{s}}} \omega_{g_{t}^{s}}^{n} \\
& \leq \int_{0}^{1} d t \int_{M}\left|\Delta\left(h_{g_{t}^{s}}-\theta_{g_{t}^{s}}\right)+X\left(h_{g_{t}^{s}}-\theta_{g_{t}^{s}}\right)+(1-s)\left(c_{1}+n\right)\right| e^{\theta_{g_{t}^{s}}} \omega_{g_{t}^{s}}^{n} \\
& +\int_{0}^{1} d t \int_{M}\left|X\left(h_{g_{t}^{s}}-\theta_{g_{t}^{s}}\right)\right| e^{\theta_{g_{t}^{s}}} \omega_{g_{t}^{s}}^{n}+V(1-s)\left(c_{1}+n\right) \\
& =\int_{0}^{1} d t \int_{M}\left|X\left(h_{g_{t}^{s}}-\theta_{g_{t}^{s}}\right)\right| e^{\theta_{g_{t}^{s}}} \omega_{g_{t}^{s}}^{n}+2 V(1-s)\left(c_{1}+n\right) \\
& \rightarrow 0, \text { as } s \rightarrow 1
\end{aligned}
$$

This finishes the proof of Lemma 4.8 .

\section{REFERENCES}

[CC] Cheeger, J. and Colding, T., On the structure of spaces with Ricci curvature bounded below I, J. Differential Geom. 45 (1997), 406-480.

[CDS] Chen, X., Donaldson, S. and Sun, S., Kähler-Einstein metrics on Fano manifolds, III, arXiv:1302.0282.

[CGT] Cheeger, J., Gromov, M. and Taylor, M., Finite propagation speed, kernel estimates for functions of the Laplace operator, and the geometry of complete Riemannian manifolds, J. Differential Geom., 17 (1982), 15-54.

[CTZ] Cao, H., Tian, G. and Zhu, X.H., Kähler-Ricci solitons on compact complex manifolds with $c_{1}(M)>0$, GAFA, Vol. 15 (2005) 697-719.

[Gr] Gross, L., Logarithmic Sobolev inequalities and contractivity properties of semigroups. Dirichlet forms (Varenna, 1992), Lecture Notes in Math., 1563 (1993), 54-88.

[Ma] Mabuchi, T., Multiplier hermitian structures on Kähler manifolds, Nagoya Math. J. 170 (2003), 73-115.

[Pe] Perelman, G., The entropy formula for the Ricci flow and its geometric applications, arXiv:math.DG/0211159.

[Li] Li, P., Lecture notes on geometric analysis, Cambridge Studies in Advanced Mathematics, No. 134 (2012), Cambridge University Press.

[Ro] Rothaus, O., Logarithmic Sobolev inequality and the spectrum of Schrödinger operators, J. Funct. Analysis, 42 (1981), no. 1, 110-120.

[T1] Tian, G., Kähler-Einstein metrics with positive scalar curvature, Invent. Math. 130 (1997), 1-37.

[T2] Tian, G., K-stability and Kähler-Einstein metrics, arXiv:1211.4669.

[TW] Tian, G. and Wang, B., On the structure of almost Einstein manifolds, arXiv: math.DG/0211159.1202.2912. 
[TZ1] Tian, G. and Zhu, X.H., Uniqueness of Kähler-Ricci soltions, Acta Math., 184 (2000), 271-305.

[TZ2] Tian, G. and Zhu, X.H., A new holomorphic invariant and uniqueness of KählerRicci solitons, Comment. Math. Helv. 77 (2002), 297-325.

[TZ3] Tian, G. and Zhu, X.H., Convergence of Kähler-Ricci flow, Journal of the Amer. Math. Soci., 20 (2007), 675-699.

[WW] Wei, G. and Wylie, W., Comparison geometry for Bakry-Emery Ricci curvature, J. Differential Geom. 83 (2009), 337-405.

[WZ] Wang, F. and Zhu, X.H., On the structure of spaces with Bakry-Émery Ricci curvature bounded below, arXiv: math.DG/1304.4490.

[Zh] Zhu, X.H., Kähler-Ricci soliton type equations on compact complex manifolds with $C_{1}(M)>0$, J. Geom. Anal., 10 (2000), 759-774.

Feng Wang, School of Mathematical Sciences, Peking University, Beijing, 100871, CHINA

Xiaohua Zhu, School of Mathematical Sciences and BiCMr, Peking UniverSity, Beijing, 100871, China, XhZHu@Math.PKu.edu.CN 